\title{
Virus del papiloma humano en carcinoma colorrectal
}

\author{
Jorge Adrián Garza-Cerna ${ }^{1}$, Carlos Pacheco-Molina ${ }^{1}$, Diana Garza-Villarreal ${ }^{1}$, \\ Arnulfo Andrei Ríos-Cantu ${ }^{1}$, Everardo Valdés-Flores ${ }^{1}$, \\ Ingrid Astrid Rodríguez-Valdés ${ }^{1}$ y Gerardo Enrique Muñoz-Maldonado ${ }^{1}$
}

\section{Human papilloma virus in colorectal carcinoma}

Introduction: Previous works have reported an association between human papilloma virus (HPV) infection and the development of colorectal cancer, and although controversy regarding this association exists. Materials and Method: This was an observational, cross-sectional, descriptive, retrospective unblinded study. Fifty pathology samples embedded in paraffin with a diagnosis of colorectal adenocarcinoma were used to isolate DNA from the tissue. DNA was extracted according to established protocols for extraction, lysis and sample rehydration. Viral DNA was identified and genotypified to amplify and detect oncogenic subtypes among 35 different subtypes included in the study, sequencing positive samples with established protocols of purification and sample analysis using microarrays. Results: Fourteen of $50(28 \%)$ samples were identified as positive for human papilloma virus; of these $11(22 \%)$ included one or more high-risk subtypes for neoplasia. Statistically significant differences were not found between the groups regarding age, sex, tumor location, degree of differentiation, infiltration, affected lymph nodes, metastasis and number of pack years. Conclusion: The detection of high-risk VPH subtypes in a high percentage of positive samples, suggests an association between infection and the development of colorectal cancer.

Key words: colorectal cancer; human papilloma virus; adenocarcinoma.

\section{Resumen}

Introducción: Previos trabajos han reportado una asociación entre la infección del virus del papiloma humano (VPH) y el desarrollo de cáncer colorrectal, aunque existe controversia al respecto. Materiales y Método: Estudio observacional, transversal, descriptivo, retrospectivo, no ciego. Se utilizaron 50 muestras de patología con diagnóstico de adenocarcinoma colorrectal, incluidas en parafina, para aislar ADN de las muestras. Se realizó la extracción de ADN mediante protocolos establecidos para extracción, lisis y rehidratación de muestra. Se identificó y genotipicó el ADN del virus para amplificar y detectar subtipos oncogénicos de entre 35 subtipos diferentes incluidos en la prueba, secuenciando las muestras positivas, utilizando protocolos ya establecidos de purificación y análisis de muestra, mediante microarreglos. Resultados: Se identificaron 14 muestras de $50(28 \%)$ estudiadas positivas para el virus de papiloma humano de las cuales $11(22 \%)$ incluyen uno o más subtipos de alto riesgo para neoplasia. No se identificaron diferencias estadísticamente significativas entre grupos en cuanto a edad, sexo, localización del tumor, grado de diferenciación, infiltración, ganglios afectados, metástasis o número de paquetes/año. Conclusión: La detección de los subtipos de VPH de alto riesgo en un alto porcentaje de las muestras positivas, sugiere una asociación entre la infección con el desarrollo de cáncer colorrectal.

Palabras clave: cáncer colorrectal; virus del papiloma humano; adenocarcinoma.

\section{Introducción}

El cáncer colorrectal (CCR) es el tipo de cáncer más común del tracto gastrointestinal, así como el tercer lugar en incidencia y mortalidad relacionada a neoplasias en nuestro continente ${ }^{1}$. Su etiología es multifactorial incluyendo dieta, ejercicio, sedenta- rismo, consumo de tabaco, uso de medicamentos, así como factores genéticos y enfermedades que afectan al tracto digestivo. Siendo la incidencia hasta 10 veces mayor en países desarrollados, asociado al estilo de vida de su población ${ }^{2}$. Se sabe que hasta un $75 \%$ de los CCR se presentan en personas sin factores de riesgo conocido, y hasta
"Hospital Universitario "Dr. José Eleuterio González", Universidad Autónoma de Nuevo León. Nuevo León México.

Recibido 2019-11-21 y aceptado 2020-02-15

Correspondencia a: Dr. Jorge Adrián Garza-Cerna jgarzauanl@gmail.com 
un $10 \%$ se asocian a entidades explicadas por la herencia genética ${ }^{2,3}$.

Sin embargo, existe un buen pronóstico de hasta un $85 \%$ de supervivencia a los 5 años del diagnóstico en pacientes con esta enfermedad que son detectados en etapas tempranas y se encuentra limitado a la pared intestinal. Esto se ve reducido conforme la enfermedad se encuentra más avanzada, y en estadios menos diferenciados, por lo cual es muy importante su correcto diagnóstico y clasificación mediante criterios histopatológicos ${ }^{4}$. Es más común, por orden de presentación, en región rectosigmoidea $(55 \%)$, ciego $(13 \%)$, colon transverso $(11 \%)$, colon ascendente $(9 \%)$ y colon descendente $(6 \%)^{5}$.

Existe información reciente que hace pensar que varios procesos neoplásicos podrían estar relacionados a patógenos. Los modelos más comúnmente asociados son los del Helicobacter pylori con cáncer gástrico y el virus de papiloma humano (VPH) con cáncer cervicouterino. Sin embargo, existen muchos otros modelos asociados a virus: herpes virus (sarcoma de Kaposi), Epstein-Barr (linfomas, cáncer nasofaríngeo) y los virus de hepatitis B y C (cáncer hepatocelular), entre otros. Se estima que hasta un 10\%-20\% de procesos neoplásicos pueden estar relacionados a infecciones por virus o bacterias ${ }^{6}$.

Respecto al VPH, se han descubierto incidencias elevadas de neoplasias asociadas a la presencia del virus, existen estudios que sugieren la posibilidad de infección de tejidos neoplásicos en colon y recto por el VPH, sugiriendo una asociación entre su presencia y el desarrollo de lesiones malignas ${ }^{7,8}$.

El virus de papiloma humano es un miembro de la familia papillomaviridae ${ }^{9}$ un grupo grande de virus pequeños, sin envoltura, con doble cadena de ácido desoxirribonucleico (ADN). Existen diversos lineamientos para identificar y clasificar a los miembros de la familia del VPH, existiendo en la actualidad más de 200, sin embargo, existen poco más de 100 bien clasificados.

Estudios recientes hablan de la probable relación entre la presencia del VPH en especímenes de CCR y el desarrollo de la neoplasia. Dichos estudios se han realizado en diferentes países, con diferencia en las incidencias reportadas desde un $2 \%$ a un $84 \%{ }^{10-16}$.

El presente estudio se diseñó pensando en determinar la prevalencia del virus del papiloma humano en especímenes de adenocarcinoma colorrectal en población mexicana, utilizando muestras obtenidas en un hospital de tercer nivel con un volumen elevado de pacientes para su estudio y con estos datos sentar una base para futuras investigaciones de esta patología y su asociación al virus.

\section{Materiales y Método}

Estudio de tipo cohorte transversal, observacional, descriptivo, retrospectivo, no ciego. En el cual se utilizaron muestras de 50 pacientes que cuentan con el diagnóstico de adenocarcinoma de colon o recto, del Hospital Universitario "Dr. José E. González", incluidas en bloques de parafina. Empleando como criterios de inclusión todos los pacientes de cualquier género, adultos, con diagnóstico de adenocarcinoma colorrectal, de quienes se cuente con espécimen de patología incluido en parafina necesario para su estudio en un periodo de 5 años previos al inicio de este estudio.

Se utilizaron datos demográficos de pacientes incluidos en el estudio, de su expediente clínico recabado durante su estancia en el Hospital Universitario "Dr. José Eleuterio González".

Se utilizan muestras de adenocarcinoma de colon y recto incluidas en bloques de parafina, se purifican y se extrae ADN de las muestras con mayor cantidad de tumor, analizando la presencia de VPH, así como su subtipo. Se procesan los resultados y se agrupan en cuanto a localización del tumor, tipo histológico y características histopatológicas, así como estadio clínico, buscando una correlación entre la presencia del virus y los hallazgos obtenidos durante el estudio de las muestras. Los microarreglos de ADN son una serie de sondas de ADN unidas a un soporte sólido en una disposición regular y prefijada. El ácido nucleico diana que será detectado puede ser ADN o ARN, y previamente a la hibridación debe ser marcado con una sustancia fluorescente o radioactiva. La principal ventaja con respecto a las técnicas de biología molecular, como la reacción en cadena de la polimerasa, es que pueden detectarse miles de genes en un mismo procesamiento. CLART ${ }^{\circledR}$ Human Papillomavirus 2 es una prueba de detección, mediante amplificación genómica, de hasta 35 genotipos de papilomavirus, a través de una plataforma de microarreglos de baja densidad para diagnóstico in vitro. Permite la detección de 35 genotipos de papilomavirus clasificados como "alto riesgo" (16, $18,26,31,33,35,39,43,45,51,52,53,56,58$, $59,66,68,70,73,82,85$ у 89$)$ y “bajo riesgo" (6, $11,40,42,44,54,61,62,71,72,81,83$ y 84), así mismo, identifica las coinfecciones presentes. Se asegura la presencia de ADN mediante un control de ADN genómico; se comprueba que la amplificación se ha realizado correctamente mediante un control interno de amplificación y todas las sondas están triplicadas, lo cual evita alteraciones locales que pudieran afectar resultados finales. La lectura 
de resultados es automática para evitar interpretaciones subjetivas, y son procesados por un lector con un software específico que automáticamente detecta e interpreta los genotipos presentes mediante la amplificación de un fragmento de 450 pares de bases que se encuentra dentro de la región L1 del virus.

El análisis estadístico se realizó mediante el programa SPSS $₫$ versión 20 , analizando y comparando las variables cualitativas mediante $\chi^{2}$, y las variables cuantitativas mediante la $\mathrm{T}$ de Student y prueba exacta de Fisher.

\section{Resultados}

Se incluyeron en el actual estudio casos de 368 pacientes del Hospital Universitario "Dr. José E. González" con diagnóstico de adenocarcinoma colorrectal, de los 368 pacientes se obtuvieron 65 casos con adecuado tejido para su estudio y expedientes clínicos completos, sin embargo, al analizar la calidad de la muestra del tejido, se redujo la cantidad a 50 muestras para su análisis. De los 50 pacientes que se estudiaron se obtuvieron 14 casos positivos para la presencia del virus de papiloma humano (28\%), 35 casos negativos $(70 \%)$ así como un caso sin $\mathrm{ADN}$ adecuado para su análisis (2\%). De las muestras positivas existen 3 con varios serotipos en la misma muestra: una muestra positiva para serotipos 16 y 31 (2\%), una para 16, 31 y $66(2 \%)$, así como una muestra positiva para 6 y $53(2 \%)$. La mayor prevalencia de un serotipo en las muestras analizadas es del serotipo 53, estando presente en un total de 9 muestras (18\%), tomando en cuenta 8 muestras con infección única por el serotipo, así como una muestra conjunta con el tipo 6, el cual tiene un porcentaje acumulado de 4 muestras (8\%) (Tabla 1).

En cuanto a la localización de las muestras de patología, encontramos que la mayoría de los casos se encuentran en recto y colon sigmoides, con una frecuencia acumulada de 28 casos $(56 \%)$, seguidos por colon ascendente con 17 casos (34\%) y colon transverso con 4 casos (8\%).

La edad de los pacientes estudiados se encuentra en un rango entre 28 y 88 años, con una media de 61,8 años, mediana de 63,5 años y una moda de 64 años. En pacientes con resultados de patología positiva para VPH, se analizaron 7 casos de individuos femeninos $(50 \%)$, así como 7 casos de individuos masculinos $(50 \%)$. La mayor cantidad de casos se encuentran en recto y colon sigmoides con 8 casos $(57,1 \%)$, seguido de colon transverso, cada uno con
Tabla 1. Frecuencia de los serotipos del VPH en muestras de tejido de pacientes con diagnóstico de adenocarcinoma rectal

\begin{tabular}{|cc|}
\hline Serotipos & Fr (\%) \\
16,31 & $1(2)$ \\
$16,31,66$ & $1(2)$ \\
6,53 & $1(2)$ \\
53 & $8(16)$ \\
6 & $3(6)$ \\
Negativo & $35(70)$ \\
No ADN & $1(2)$ \\
Total & $50(100)$ \\
\hline
\end{tabular}

$\mathrm{Fr}=$ Frecuencia

Tabla 2. Regiones del colon con CCR

\begin{tabular}{|lrrrr|}
\hline Localización & Fr CCR (\%) & Fr VPH [+] \\
Recto y sigmoides & 28 & $(56)$ & 8 & $(57,1)$ \\
Colon derecho & 17 & $(34)$ & 3 & $(21,5)$ \\
Colon transverso & 4 & $(8)$ & 3 & $(21,4)$ \\
Colon descendente & 1 & $(2)$ & & \\
Total & $50(100)$ & $14(100)$ \\
\hline
\end{tabular}

$\mathrm{Fr}=$ Frecuencia

3 casos $(21,4 \%)$, hallando prevalencia de este en colon ascendente (Tabla 2).

Al analizar comparativamente las muestras, encontramos que no hay diferencia estadísticamente significativa entre el sexo de pacientes entre grupos, la localización del tumor, el tipo histológico, grado de diferenciación, nivel de infiltración, afección ganglionar, metástasis y estadio clínico (Tabla 3).

\section{Discusión}

Desde los primeros informes sobre el estudio de la presencia y propagación del virus del papiloma humano en el cáncer colorrectal, expresados a fines de la década de $1980^{19}$, se han realizado una gran cantidad de estudios; sin embargo, las tasas de prevalencia del VPH en el CCR varían según los datos disponibles, que van del $0 \%$ al $85 \%{ }^{20}$.

La participación del virus del papiloma humano en la carcinogénesis del cáncer colorrectal es un tema polémico. Ibragimova et al. ${ }^{21}$, realizaron un 


\section{ARTÍCULO ORIGINAL}

Tabla 3. Comparación entre grupos (positivo y negativo para VPH)

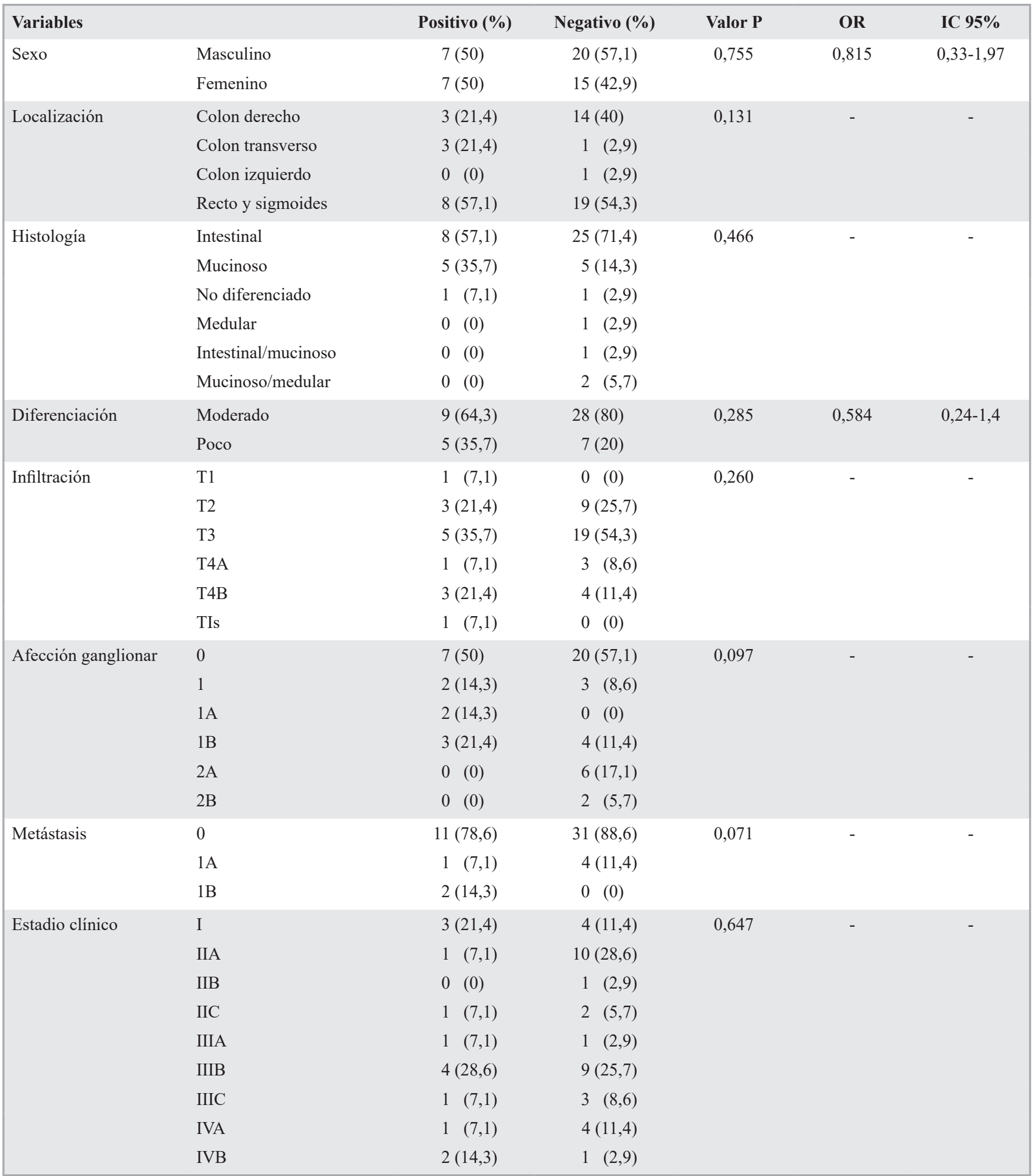


metaanálisis para sistematizar los resultados de investigación actualmente disponibles sobre el tema. El análisis se basó en los datos de 19 estudios para evaluar la asociación de la infección por VPH con el cáncer colorrectal. Según los datos obtenidos, determinaron que el nivel estadísticamente significativo de infección por VPH en el tejido tumoral de CCR y el riesgo relativo resultante de desarrollar CCR con infección por VPH es RR (IC 95\%) = 2,97 (1,42$6,22) \operatorname{con} \mathrm{p}=0,0039$.

En base a nuestro estudio se logró la identificación de hasta un $28 \%$ de muestras positivas para VPH, lo cual se encuentra muy cerca del promedio de detección publicado en un metaanálisis realizado por Damin et al. ${ }^{17}$, en el cual es de un $31,9 \%$ de prevalencia general; destacando en nuestra muestra el serotipo 53 con una mayor prevalencia, incluido en el grupo de alto riesgo, lo cual difiere de los estudios previos realizados sobre este tema, sin embargo, una probable razón es que en la mayoría de estudios publicados no se ha incluido este serotipo para su estudio $^{8,14,17}$. Existe una falta de estudios prospectivos bien planeados para valorar con suficiente seguridad la relación entre el virus y carcinogénesis en adenocarcinoma colorrectal, sin embargo, hay suficiente información disponible que sugiere una relación causa-efecto ${ }^{14,17}$. De las muestras positivas cabe destacar que se encontraron los serotipos 6,16 , 31,53 y 66; de los cuales, los serotipos 16, 31, 53 y 66 son considerados de alto riesgo para neoplasia. Logramos identificar en un alto porcentaje de especímenes, un serotipo raramente incluido en otros estudios, el cual normalmente no se incluye, por ser tan poco prevalente en otros tipos de cáncer como el cervicouterino ${ }^{18}$.

Consideramos de manera oportuna el destacar el panorama epidemiológico de esta asociación, Damin et al. ${ }^{17}$, identifican una prevalencia menor en Europa con un 14,1\% y siendo la región con una mayor prevalencia América del Sur con un $60,8 \%$. El serotipo 18 fue el virus más frecuente en los casos de cáncer colorrectal en la región de Asia con un 73,34\% y en Europa con un 47,3\%; en contraste el serotipo 16 fue más frecuente en tumores colorrectales de América del Sur con un 58,3\%. Así como el resto la bibliografía referente a este tema lo ha descrito, como es el caso de Pelizzer et al. ${ }^{22}$, donde se realiza una evaluación sistemática de múltiples de la asociación VPH con CCR donde se identifica una prevalencia en dos serotipos en particular, el 16 con un $31,7 \%$ y el 18 con $35,8 \%$, destacando una asociación similar.

En cuanto a la localización de los tumores evaluados, se cuenta con un promedio muy pare- cido al incluido en la literatura mundial, con un porcentaje mayor en región rectosigmoidea. En orden descendente de prevalencia se encuentra el colon ascendente, ciego y colon transverso, con porcentajes muy parecidos a lo publicado ${ }^{5}$ En caso de tumores en región rectosigmoidea podría encontrarse una asociación entre el sitio tan cercano a región anogenital y la infección, sin embargo, en sitios alejados como el ciego, colon ascendente y colon transverso, cabría la posibilidad de identificar alguna otra vía de contagio y diseminación del virus.

Hablando de los aspectos demográficos y clínicos, no se hallaron diferencias significativas en cuanto a edad, sexo, localización del tumor, grado de diferenciación, infiltración, ganglios afectados o metástasis entre los grupos positivo y negativo, lo cual corresponde a otros estudios donde tampoco se encontraron diferencias.

Existen diversos métodos de identificación del virus, lo cual podría ser responsable de las diferencias en resultados obtenidos en estudios publicados previamente, así como la calidad del ADN de las muestras analizadas. Sería importante desarrollar un protocolo de estudio, para llegar a un método de detección adecuado y lograr la correcta valoración de las muestras en diferentes centros de estudio. Así mismo, logramos identificar en un alto porcentaje de especímenes, un serotipo raramente incluido en otros estudios, el cual normalmente no se incluye en los serotipos a estudiar, por ser tan poco prevalente en otros tipos de cáncer como el cervicouterino $^{18}$.

\section{Conclusión}

En conclusión, los resultados de nuestro estudio apoyan la teoría de una asociación entre adenocarcinoma colorrectal y el VPH, sin embargo, existe la necesidad de estudios mejor diseñados para definir una correlación causal entre la presencia de cáncer y la infección de tejido. Estudios prospectivos con un mayor número de pacientes podrían aportar más información sobre el tema, así como identificar diferencias estadísticamente significativas entre ambos grupos, las cuales no pueden ser adecuadamente valoradas con una población tan reducida de pacientes.

No hay otros estudios en población mexicana que valoren este fenómeno, lo cual abre la puerta a nuevas hipótesis y a la necesidad de recabar mayor cantidad de información en poblaciones con esta patología, así como también ofrece información útil 
que crea una pauta para incluir en nuevos estudios una mayor cantidad de serotipos, para evaluar la prevalencia real de VPH en especímenes de adenocarcinoma colorrectal. Es importante definir nuevos protocolos de estudio para estandarizar métodos de detección, así como estudiar nuevas formas de diseminación del virus para explicar tumoraciones tan alejadas de un probable sitio de entrada, como sería la región anogenital.

\section{Responsabilidades éticas}

Protección de personas y animales. Los autores declaran que para esta investigación no se han realizado experimentos en seres humanos ni en animales.

Confidencialidad de los datos. Los autores declaran que en este artículo no aparecen datos de pacientes.

Conflictos de interés: no hay.

\section{Bibliografía}

1. PAHO/WHO. Colorectal cancer in the Americas. Washington, D.C. 2014.

2. Morgan G, Silva AM, Sat D. Factores de riesgo para cáncer colorrectal. GAMO 2008;7:12-15.

3. Schlussel AT, Gagliano RA Jr, SetoDonlon S, Eggerding F, Donlon T, Berenberg J, et al. The evolution of colorectal cancer genetics-Part 1: from discovery to practice. J Gastrointest Oncol. 2014;5:326-35. doi: 10.3978/j. issn.2078-6891.2014.069.

4. Herrera R. Carcinoma de colon y recto. Diagnóstico histológico y estadificación. GAMO 2008;7:22-30.

5. Winawer SJ, Fletcher RH, Miller L, Godlee F, Stolar MH, Mulrow CD, et al. Colorectal cancer screening: clinical guidelines and rationale. Gastroenterology 1997;112:594-642. doi: 10.1053/ gast.1997.v112.agast970594.

6. Selgrad M, Malfertheiner P, Fini L, Goel A, Boland CR, Ricciardiello L. The role of viral and bacterial pathogens in gastrointestinal cancer. J Cell Physiol. 2008;216:378-88. doi: 10.1002/jcp.21427.

7. Pérez LO, Abba MC, Laguens RM, Golijow CD. Analysis of adenocarcinoma of the colon and rectum: detection of human papillomavirus (HPV) DNA by polymerase chain reaction. Colorectal Dis. 2005; 7:492-5. doi:10.1111/j.14631318.2005.00774.X

8. Liu F, Mou X, Zhao N, Lin J, Teng L, Xiang C. Prevalence of human papillomavirus in Chinese patients with colorectal cancer. Colorectal Dis 2011;13:865-71. doi: 10.1111/j.14631318.2010.02335.x.

9. McLaughlin-Drubin ME, Meyers J, Munger K. Cancer associated human papillomaviruses. Curr Opin Virol. 2012;2:459-66. doi: 10.1016/j. coviro.2012.05.004.

10. Burnett-Hartman AN, Feng Q, Popov V, Kalidindi A, Newcomb PA. Human papillomavirus DNA is rarely detected in colorectal carcinomas and not associated with microsatellite instability: the Seattle colon cancer family registry. Cancer Epidemiol Biomarkers Prev. 2013;22:31719. doi: 10.1158/1055-9965.EPI-12-1170.

11. Raj GV, Moreno JG, Gomella LG. Utilization of polymerase chain reaction technology in the detection of solid tumors. Cancer 1998;82:141942. doi: 10.1002/(sici)10970142(19980415)82:8<1419::aidcncr1>3.0.co;2-4.

12. Buyru N, Tezol A, Dalay N. Coexistence of K-ras mutations and HPV infection in colon cancer. BMC Cancer 2006;6:115. doi: 10.1186/1471-2407-6-115.

13. Cheng JY, Sheu LF, Meng CL, Lee WH, Lin JC. Detection of human papillomavirus DNA in colorectal carcinomas by polymerase chain reaction. Gut 1995;37:87-90. doi: 10.1136/ gut.37.1.87.

14. Lorenzon L, Ferri M, Pilozzi E, Torrisi MR, Ziparo V, French D. Human papillomavirus and colorectal cancer: evidences and pitfalls of published literature. Int J Colorectal Dis. 2011;26:135-142. doi: 10.1007/s00384010-1049-8.

15. Pérez LO, Barbisan G, Ottino A, Pianzola $\mathrm{H}$, Golijow CD. Human papillomavirus DNA and oncogene alterations in colorectal tumors. Pathol Oncol Res. 2010; 16 (3): 461-8. doi: 10.1007/s12253010-9246-x

16. Picanço-Junior OM, Oliveira AL, Freire LT, Brito RB, Villa LL, Matos
D. Association between human

Papillomavirus and colorectal adenocarcinoma and its influence on tumor staging and degree of cell differentiation. Arq Bras Cir Dig. 2014;27:172-6. doi: 10.1590/s010267202014000300003.

17. Damin DC, Ziegelmann PK, Damin AP. Human papillomavirus infection and colorectal cancer risk: a meta-analysis. Colorectal Dis. 2013;15:e420-e428. doi: 10.1111/codi.12257.

18. Muñoz N, Bosch FX, de Sanjosé S, Herrero R, Castellsagué X, Shah KV, et al. Epidemiologic classification of human papillomavirus types associated with cervical cancer. N Engl J Med. 2003;348:518-27. doi: 10.1056/ NEJMoa021641.

19. Beckmann AM, Daling JR, Sherman KJ, Maden C, Miller BA, Coates RJ, et al. Human papillomavirus infection and anal cancer. Int J Cancer. 1989;43:1042-9. doi:10.1002/ijc.2910430615.

20. Lee YM, Leu SY, Chiang H, Fung CP, Liu WT. Human papillomavirus type 18 in colorectal cancer. J Microbiol Immunol Infect. 2001;34:87-91.

21. Ibragimova MK, Tsyganov MM, Litviakov NV. Human papillomavirus and colorectal cancer. Med Oncol. 2018;35:140. doi: 10.1007/s12032-0181201-9.

22. Pelizzer T, Dias CP, Poeta J, Torriani T, Roncada C. Colorectal cancer prevalence linked to human papillomavirus: a systematic review with meta-analysis. Prevalência de câncer colorretal associado ao papilomavírus humano: uma revisão sistemática com metanálise. Rev Bras Epidemiol. 2016;19:791-802. doi: 10.1590/19805497201600040009 . 